\title{
Factorization of Cesàro operator and related inequalities
}

Hadi Roopaei ${ }^{\text {* }^{*}}$

\section{"Correspondence:}

h.roopaei@gmail.com

${ }^{1}$ Department of Mathematics and

Statistics, University of Victoria,

Victoria, Canada

\section{Springer}

\begin{abstract}
In this paper, we introduce two factorizations for the Cesàro matrix of order $n$ based on Cesàro and gamma matrices. The results of these factorizations are new inequalities, one of which is a generalized version of the well-known Hardy's inequality. Moreover, we obtain the norm of Cesàro operator of order $n$ on Cesàro and gamma matrix domains.
\end{abstract}

MSC: 46B45; 46A45; 40G05; 47B37

Keywords: Cesàro matrix; Hausdorff matrix; Hilbert matrix; Sequence space

\section{Introduction}

Let $\omega$ denote the vector space of all real-valued sequences $\mathbf{x}=\left(x_{k}\right)_{k=0}^{\infty}$. Any linear subspace of $\omega$ is called a sequence space. In particular, the Banach space $\ell_{p}$ is the set of all $\mathbf{x} \in \omega$ such that

$$
\|\mathbf{x}\|_{\ell_{p}}=\left(\sum_{k=0}^{\infty}\left|x_{k}\right|^{p}\right)^{1 / p}<\infty, \quad 1 \leq p<\infty .
$$

In our discussion, we consider infinite matrices $A=\left(a_{i, j}\right)$, where the indices $i$ and $j$ run through $\mathbb{N}_{0}:=\{0,1,2, \ldots\}$. The matrix domain of $A$, with respect to a fixed sequence space $\mathcal{X}$, is defined as

$$
A(\mathcal{X})=\{\mathbf{x} \in \omega: A \mathbf{x} \in \mathcal{X}\} .
$$

In the special but important case $\mathcal{X}=\ell_{p}$, we will simply write $A(p)$ instead of $A\left(\ell_{p}\right)$. Some further special notations are described below. It is rather trivial that $I(p)=\ell_{p}$, where $I$ is the infinite identity matrix. This concept has inspired many researchers to seek and define new Banach spaces as the domain of an infinite matrix.

The operator $T$ is called bounded if the inequality $\|T x\|_{\ell_{p}} \leq K\|x\|_{\ell_{p}}$ holds for all sequences $x \in \ell_{p}$, while the constant $K$ does not depend on $x$. The constant $K$ is called an upper bound for operator $T$, and the smallest possible value of $K$ is called the norm of $T$. One of the advantages of finding the norm of an operator is obtaining useful inequalities.

(c) The Author(s) 2021. This article is licensed under a Creative Commons Attribution 4.0 International License, which permits use, sharing, adaptation, distribution and reproduction in any medium or format, as long as you give appropriate credit to the original author(s) and the source, provide a link to the Creative Commons licence, and indicate if changes were made. The images or other third party material in this article are included in the article's Creative Commons licence, unless indicated otherwise in a credit line to the material. If material is not included in the article's Creative Commons licence and your intended use is not permitted by statutory regulation or exceeds the permitted use, you will need to obtain permission directly from the copyright holder. To view a copy of this licence, visit http://creativecommons.org/licenses/by/4.0/. 
The well-known inequalities

$$
\sum_{n=0}^{\infty}\left(\sum_{k=0}^{n} \frac{\left|x_{k}\right|}{n+1}\right)^{p} \leq\left(\frac{p}{p-1}\right)^{p} \sum_{k=0}^{\infty}\left|x_{k}\right|^{p}
$$

and

$$
\sum_{n=0}^{\infty}\left(\sum_{k=0}^{\infty} \frac{\left|x_{k}\right|}{n+k+1}\right)^{p} \leq(\pi \csc (\pi / p))^{p} \sum_{k=0}^{\infty}\left|x_{k}\right|^{p},
$$

which are also known as Hardy's inequality and Hilbert's inequality, have resulted from the boundedness of Cesàro and Hilbert operators.

The problem of finding norms of operators has been investigated in some of the following references $[7,9-13,15,17,18]$.

The organization of paper is as follows. In Sect. 2, we introduce the family of Hausdorff matrices $H^{\mu}$. The definition is very general and includes several important subfamilies, e.g., Cesàro and gamma matrices. We also introduce the classical Hilbert matrix in this section.

In Sect. 3, we state our main theorem, where we introduce two factorizations for the Cesàro matrix, which results in some interesting inequalities. The other result is obtaining a factorization for the Hilbert operator based on the Cesàro matrix, which the author had introduced before in [13, Corollary 2.3], but with a new approach.

In Sect. 4, as an application of those introduced factorizations for the Cesàro matrix, we compute the norm of Cesàro operator of order $n$ from the Cesàro and gamma sequence spaces into the $\ell_{p}$ space.

\section{The Cesàro and gamma matrices}

Let $\mu$ be a probability measure on $[0,1]$. The Hausdorff matrix $H^{\mu}=\left(h_{j, k}\right)_{j, k=0}^{\infty}$ is an infinite matrix with the entries

$$
h_{j, k}=\left(\begin{array}{l}
j \\
k
\end{array}\right) \int_{0}^{1} \theta^{k}(1-\theta)^{j-k} d \mu(\theta), \quad 0 \leq k \leq j,
$$

and $h_{j, k}=0$ for $k>j$. As a direct consequence of Hardy's formula [6, Theorem 216], we know that the Hausdorff matrix is a bounded operator on $\ell_{p}, 1 \leq p<\infty$, if and only if

$$
\int_{0}^{1} \theta^{\frac{-1}{p}} d \mu(\theta)<\infty
$$

moreover,

$$
\left\|H^{\mu}\right\|_{\ell_{p} \rightarrow \ell_{p}}=\int_{0}^{1} \theta^{\frac{-1}{p}} d \mu(\theta) .
$$

Bennett $[1,3]$ extensively studied these matrices. He showed that Hausdorff operators have the norm separating property [2, Theorem 9]. More explicitly, if $p \geq 1$ and $H^{\mu}, H^{\varphi}$, and $H^{v}$ are Hausdorff matrices such that $H^{\mu}=H^{\varphi} H^{v}$, then $H^{\mu}$ is bounded on $\ell_{p}$ if and only if both $H^{\varphi}$ and $H^{v}$ are bounded on $\ell_{p}$. Moreover, we have

$$
\left\|H^{\mu}\right\|_{\ell_{p} \rightarrow \ell_{p}}=\left\|H^{\varphi}\right\|_{\ell_{p} \rightarrow \ell_{p}}\left\|H^{v}\right\|_{\ell_{p} \rightarrow \ell_{p}} .
$$


The Hausdorff matrix contains several famous classes of matrices as a special case. We mention a few examples below. In all cases, we assume that $n>0$. We also denote the conjugate exponent of $p$ by $p^{*}$, i.e., $1 / p+1 / p^{*}=1$.

We use the notation $\operatorname{hau}(p)$ as the set of all sequences whose $H^{\mu}$-transforms are in the space $\ell_{p}$, that is,

$$
\operatorname{hau}(p)=\left\{x=\left(x_{j}\right) \in \omega: \sum_{j=0}^{\infty}\left|\sum_{k=0}^{j} \int_{0}^{1}\left(\begin{array}{l}
j \\
k
\end{array}\right) \theta^{k}(1-\theta)^{j-k} d \mu(\theta) x_{k}\right|^{p}<\infty\right\},
$$

where $\mu$ is a fixed probability measure on $[0,1]$.

Cesàro matrix of order $n$. The measure

$$
d \mu(\theta)=n(1-\theta)^{n-1} d \theta
$$

gives the Cesàro matrix $C^{n}=\left(C_{j, k}^{n}\right)$ of order $n$, for which

$$
C_{j, k}^{n}= \begin{cases}\frac{\left(\begin{array}{c}
n+j-k-1 \\
j-k
\end{array}\right)}{\left(\begin{array}{c}
n+j \\
j
\end{array}\right)}, & 0 \leq k \leq j \\
0, & \text { otherwise. }\end{cases}
$$

Hence, according to (2.1), $C^{n}$ has the $\ell_{p}$-norm

$$
\left\|C^{n}\right\|_{\ell_{p} \rightarrow \ell_{p}}=\frac{\Gamma(n+1) \Gamma\left(1 / p^{*}\right)}{\Gamma\left(n+1 / p^{*}\right)} .
$$

Note that $C^{0}=I$, where $I$ is the identity matrix, and

$$
C^{1}=C=\left(\begin{array}{cccc}
1 & 0 & 0 & \ldots \\
1 / 2 & 1 / 2 & 0 & \ldots \\
1 / 3 & 1 / 3 & 1 / 3 & \ldots \\
\vdots & \vdots & \vdots & \ddots
\end{array}\right)
$$

is the classical Cesàro matrix, which has the $\ell_{p}$-norm $\|C\|_{\ell_{p} \rightarrow \ell_{p}}=p^{*}$. According to (1.1), the matrix domain of $C^{n}$ is

$$
C^{n}(p)=\left\{\mathbf{x} \in \omega: \sum_{j=0}^{\infty}\left|\frac{1}{\left(\begin{array}{c}
n+j \\
j
\end{array}\right)} \sum_{k=0}^{j}\left(\begin{array}{c}
n+j-k-1 \\
j-k
\end{array}\right) x_{k}\right|^{p}<\infty\right\} .
$$

Equipped with the norm

$$
\|\mathbf{x}\|:=\left(\sum_{j=0}^{\infty}\left|\frac{1}{\left(\begin{array}{c}
n+j \\
j
\end{array}\right)} \sum_{k=0}^{j}\left(\begin{array}{c}
n+j-k-1 \\
j-k
\end{array}\right) x_{k}\right|^{p}\right)^{1 / p}
$$

$C^{n}(p)$ is a Banach space. In literature, $\operatorname{ces}(n, p)$ is also used to denote this space [3]. The matrix $C$ was defined by Ernesto Cesàro (1859-1906), an Italian mathematician who worked in the field of differential geometry. Since then, many mathematicians have studied Cesàro 
sequence and Cesàro function spaces. However, the main focus has been on the Cesàro matrix of order one. The Cesàro sequence space $C^{1}(p)$, its topological properties, its matrix transformations, and matrix domains are studied in $[8,16]$. Recently, the general case $C^{n}(p)$ and its topological properties, matrix transformations, and matrix domains for $0<p<\infty$ have been thoroughly studied $[14,15]$.

Gamma matrix of order $n$. The measure

$$
d \mu(\theta)=n \theta^{n-1} d \theta
$$

gives the gamma matrix $\Gamma^{n}=\left(\gamma_{j, k}^{n}\right)$ of order $n$, for which

$$
\gamma_{j, k}^{n}= \begin{cases}\frac{\left(\begin{array}{c}
n+k-1 \\
k
\end{array}\right)}{\left(\begin{array}{c}
(+j \\
j
\end{array}\right)}, & 0 \leq k \leq j \\
0, & \text { otherwise. }\end{cases}
$$

Hence, according to (2.1), $\Gamma^{n}$ has the $\ell_{p}$-norm

$$
\left\|\Gamma^{n}\right\|_{\ell_{p} \rightarrow \ell_{p}}=\frac{n p}{n p-1}
$$

While $\Gamma^{1}$ is the classical Cesàro matrix $C$, other values of $n$ give birth to different matrices. For example,

$$
\Gamma^{2}=\left(\begin{array}{cccc}
1 & 0 & 0 & \ldots \\
1 / 3 & 2 / 3 & 0 & \ldots \\
1 / 6 & 2 / 6 & 3 / 6 & \ldots \\
\vdots & \vdots & \vdots & \ddots
\end{array}\right) \text { and } \Gamma^{3}=\left(\begin{array}{cccc}
1 & 0 & 0 & \ldots \\
1 / 4 & 3 / 4 & 0 & \ldots \\
1 / 10 & 3 / 10 & 6 / 10 & \ldots \\
\vdots & \vdots & \vdots & \ddots
\end{array}\right)
$$

According to (1.1), the matrix domain of $\Gamma^{n}$ is

$$
\Gamma^{n}(p)=\left\{x=\left(x_{k}\right) \in \omega: \sum_{j=0}^{\infty}\left|\frac{1}{\left(\begin{array}{c}
n+j \\
j
\end{array}\right)} \sum_{k=0}^{j}\left(\begin{array}{c}
n+k-1 \\
k
\end{array}\right) x_{k}\right|^{p}<\infty\right\},
$$

which equipped with the norm

$$
\|\mathbf{x}\|:=\left(\sum_{j=0}^{\infty}\left|\frac{1}{\left(\begin{array}{c}
n+j \\
j
\end{array}\right)} \sum_{k=0}^{j}\left(\begin{array}{c}
n+k-1 \\
k
\end{array}\right) x_{k}\right|^{p}\right)^{\frac{1}{p}}
$$

becomes a Banach space. This space is also denoted by $\operatorname{gam}(n, p)$.

Hilbert matrix. In this study, we also need the Hilbert matrix $\mathfrak{H}=\left(h_{j, k}\right)$, which is defined by

$$
h_{j, k}=\frac{1}{j+k+1}, \quad j, k \geq 0 .
$$


More explicitly,

$$
\mathfrak{H}=\left(\begin{array}{cccc}
1 & 1 / 2 & 1 / 3 & \ldots \\
1 / 2 & 1 / 3 & 1 / 4 & \ldots \\
1 / 3 & 1 / 4 & 1 / 5 & \ldots \\
\vdots & \vdots & \vdots & \ddots
\end{array}\right) \text {. }
$$

This matrix was introduced by David Hilbert in 1894 to study a question in approximation theory.

\section{Main theorem}

In this section, factorization of the Cesàro operator based on Cesàro and gamma matrices is investigated. First, we need some preliminaries. For nonnegative integers $n, j$, and $k$, let us define the matrix $B^{n}=\left(b_{j, k}^{n}\right)$ by

$$
\begin{aligned}
b_{j, k}^{n} & =\frac{(k+1) \cdots(k+n)}{(j+k+1) \cdots(j+k+n+1)} \\
& =\left(\begin{array}{c}
n+k \\
k
\end{array}\right) \beta(j+k+1, n+1) \quad(j, k=0,1, \ldots),
\end{aligned}
$$

where $\beta$ is the beta function

$$
\beta(m, n)=\int_{0}^{1} z^{m-1}(1-z)^{n-1} d z, \quad m, n=1,2, \ldots
$$

Note that, for $n=0, B^{0}=\mathfrak{H}$, where $\mathfrak{H}$ is the Hilbert matrix. We know that $B^{n}$ is a bounded operator on $\ell_{p}$ and

$$
\left\|B^{n}\right\|_{\ell_{p} \rightarrow \ell_{p}}=\frac{\Gamma\left(n+1 / p^{*}\right) \Gamma(1 / p)}{\Gamma(n+1)} .
$$

See [10, Lemma 2.3].

In the next result, we reveal the relation between the Cesàro and gamma matrices which have the same entries in a row, but the inverse order in columns. Moreover, we introduce some factorizations for the Cesàro matrix based on Cesàro and gamma matrices, which results in some interesting inequalities and inclusions.

Theorem 3.1 Let $n \geq m \geq 1$, and let $C^{n}$ and $\Gamma^{m}$ be respectively the Cesàro and gamma matrices of order $n$ and $m$. Then the following assertions hold:

(a) $C^{n}=C^{n-1} \Gamma^{n}$.

(b) $C^{n}=\Gamma^{1} \Gamma^{2} \ldots \Gamma^{n}$.

(c) $C^{n}=R^{n, m} \Gamma^{m}$, where

$$
R^{n, m}=\prod_{i=1, i \neq m}^{n} \Gamma^{i}
$$

Moreover, $R^{n, m}$ is a bounded operator on $\ell_{p}$ with the norm

$$
\left\|R^{n, m}\right\|_{\ell_{p} \rightarrow \ell_{p}}=\frac{(1-1 / m p) \Gamma(n+1) \Gamma\left(1 / p^{*}\right)}{\Gamma\left(n+1 / p^{*}\right)} .
$$


(d) $C^{n}=S^{n, m} C^{m}$, where

$$
S^{n, m}=\prod_{i=m+1}^{n} \Gamma^{i}
$$

Moreover, $S^{n, m}$ is a bounded operator on $\ell_{p}$ with the norm

$$
\left\|S^{n, m}\right\|_{\ell_{p} \rightarrow \ell_{p}}=\frac{\Gamma(n+1) \Gamma\left(m+1 / p^{*}\right)}{\Gamma(m+1) \Gamma\left(n+1 / p^{*}\right)} .
$$

Proof (a): Since Hausdorff matrices commute, by using the identity

$$
\sum_{k=0}^{j}\left(\begin{array}{c}
n+j-k-1 \\
j-k
\end{array}\right)=\sum_{k=0}^{j}\left(\begin{array}{c}
n+k-1 \\
k
\end{array}\right)=\left(\begin{array}{c}
n+j \\
j
\end{array}\right)
$$

we have

$$
\begin{aligned}
\left(\Gamma^{n} C^{n-1}\right)_{i, k} & =\sum_{j=k}^{i} \Gamma_{i, j}^{n} C_{j, k}^{n-1}=\sum_{j=k}^{i} \frac{\left(\begin{array}{c}
n+j-1 \\
j
\end{array}\right)}{\left(\begin{array}{c}
n+i \\
i
\end{array}\right)} \frac{\left(\begin{array}{c}
n+j-k-2 \\
j-k
\end{array}\right)}{\left(\begin{array}{c}
n+j-1 \\
j
\end{array}\right)} \\
& =\frac{1}{\left(\begin{array}{c}
n+i \\
i
\end{array}\right)} \sum_{j=k}^{i}\left(\begin{array}{c}
n+j-k-2 \\
j-k
\end{array}\right) \\
& =\frac{1}{\left(\begin{array}{c}
n+i \\
i
\end{array}\right)} \sum_{j=0}^{i-k}\left(\begin{array}{c}
n+j-2 \\
j
\end{array}\right)=\frac{\left(\begin{array}{c}
n+i-k-1 \\
i-k
\end{array}\right)}{\left(\begin{array}{c}
n+i \\
i
\end{array}\right)}=C_{i, k}^{n} .
\end{aligned}
$$

Therefore $C^{n}=C^{n-1} \Gamma^{n}$.

(b): This is a direct consequence of $(a)$.

$(c)$ : According to part (a) and by induction,

$$
C^{n}=\Gamma^{1} \ldots \Gamma^{m-1} \Gamma^{m+1} \ldots \Gamma^{n} \times \Gamma^{m}=R^{n, m} \Gamma^{m} .
$$

Now, according the norm separating property of Hausdorff matrices (2.2), we have

$$
\begin{aligned}
\left\|R^{n, m}\right\|_{\ell_{p} \rightarrow \ell_{p}} & =\left\|\Gamma^{1}\right\|_{\ell_{p} \rightarrow \ell_{p}} \cdots\left\|\Gamma^{m-1}\right\|_{\ell_{p} \rightarrow \ell_{p}}\left\|\Gamma^{m+1}\right\|_{\ell_{p} \rightarrow \ell_{p}} \cdots\left\|\Gamma^{n}\right\|_{\ell_{p} \rightarrow \ell_{p}} \\
& =\frac{\prod_{i=1}^{n}\left\|\Gamma^{i}\right\|_{\ell_{p} \rightarrow \ell_{p}}}{\left\|\Gamma^{m}\right\|_{\ell_{p} \rightarrow \ell_{p}}} \\
& =\frac{\left\|C^{n}\right\|_{\ell_{p} \rightarrow \ell_{p}}}{\left\|\Gamma^{m}\right\|_{\ell_{p} \rightarrow \ell_{p}}} \\
& =\frac{(1-1 / m p) \Gamma(n+1) \Gamma\left(1 / p^{*}\right)}{\Gamma\left(n+1 / p^{*}\right)} .
\end{aligned}
$$

$(d)$ : According to part $(b)$, we have $C^{n}=C^{m} \Gamma^{m+1} \ldots \Gamma^{n}$. Hence, by the definition $S^{n, m}=$ $\Gamma^{m+1} \ldots \Gamma^{n}$, we have the claimed factorization. For computing the norm of $S^{n, m}$, note that

$$
\left\|S^{n, m}\right\|_{\ell_{p} \rightarrow \ell_{p}}=\frac{\left\|C^{n}\right\|_{\ell_{p} \rightarrow \ell_{p}}}{\left\|C^{m}\right\|_{\ell_{p} \rightarrow \ell_{p}}}=\frac{\Gamma(n+1) \Gamma\left(m+1 / p^{*}\right)}{\Gamma(m+1) \Gamma\left(n+1 / p^{*}\right)} .
$$


Bennett found the corresponding measure $d \mu(\theta)$ for the matrix $S^{m, n}=\Gamma^{m+1} \cdots \Gamma^{n}$ which we obtained through Theorem 3.1. He showed

$$
d \mu(\theta)=\frac{\Gamma(n+1)}{\Gamma(m+1) \Gamma(n-m)} \theta^{m}(1-\theta)^{n-m-1} d \theta .
$$

The matrix $S^{n, m}=\left(s_{j, k}^{n, m}\right)$ has the entries

$$
s_{j, k}^{n, m}= \begin{cases}\frac{\left(\begin{array}{c}
m+k \\
k
\end{array}\right)\left(\begin{array}{c}
n-m+j-k-1 \\
j-k
\end{array}\right)}{\left(\begin{array}{c}
n+j \\
j
\end{array}\right)}, & j \geq k \geq 0 \\
0, & \text { otherwise. }\end{cases}
$$

See $[1,2]$.

We can rewrite the factorization discussed in Theorem 3.1 as

$$
\begin{aligned}
\left\|C^{n} x\right\|_{\ell_{p}} & \leq\left\|C^{n-1}\right\|_{\ell_{p} \rightarrow \ell_{p}}\left\|\Gamma^{n} x\right\|_{\ell_{p}} \\
\left\|C^{n} x\right\|_{\ell_{p}} & \leq\left\|\Gamma^{n}\right\|_{\ell_{p} \rightarrow \ell_{p}}\left\|C^{n-1} x\right\|_{\ell_{p}} \\
\left\|C^{n} x\right\|_{\ell_{p}} & \leq\left\|R^{n, m}\right\|_{\ell_{p} \rightarrow \ell_{p}}\left\|\Gamma^{m} x\right\|_{\ell_{p}} \\
\left\|C^{n} x\right\|_{\ell_{p}} & \leq\left\|S^{n, m}\right\|_{\ell_{p} \rightarrow \ell_{p}}\left\|C^{m} x\right\|_{\ell_{p}}
\end{aligned}
$$

In the first place, these inequalities imply

- $\Gamma^{n}(p) \subset C^{n}(p)$,

- $C^{n-1}(p) \subset C^{n}(p)$,

- $\Gamma^{m}(p) \subset C^{n}(p)$

- $C^{m}(p) \subset C^{n}(p)$,

where $n \geq m \geq 1$. Secondly, combined with (2.4) and (3.3), we express a more explicit account of the above inequalities.

Corollary 3.2 Let $\left(x_{n}\right)$ be a sequence of real numbers. Then the following statements hold.

(a) For $n \geq 1$,

$$
\sum_{j=0}^{\infty}\left|\sum_{k=0}^{j} \frac{\left(\begin{array}{c}
n+j-k-1 \\
j-k
\end{array}\right)}{\left(\begin{array}{c}
n+j \\
j
\end{array}\right)} x_{k}\right|^{p} \leq\left(\frac{n p}{n p-1}\right)^{p} \sum_{j=0}^{\infty}\left|\sum_{k=0}^{j} \frac{\left(\begin{array}{c}
n+j-k-2 \\
j-k
\end{array}\right)}{\left(\begin{array}{c}
n+j-1 \\
j
\end{array}\right)} x_{k}\right|^{p}
$$

In particular, for $n=1$, we have Hardy's inequality

$$
\sum_{j=0}^{\infty}\left|\sum_{k=0}^{j} \frac{x_{k}}{1+j}\right|^{p} \leq\left(\frac{p}{p-1}\right)^{p} \sum_{j=0}^{\infty}\left|x_{k}\right|^{p}
$$

(b) For $n \geq m \geq 1$,

$$
\sum_{j=0}^{\infty}\left|\sum_{k=0}^{j} \frac{\left(\begin{array}{c}
n+j-k-1 \\
j-k
\end{array}\right)}{\left(\begin{array}{c}
n+j \\
j
\end{array}\right)} x_{k}\right|^{p} \leq\left[\frac{(1-1 / m p) \Gamma(n+1) \Gamma\left(1 / p^{*}\right)}{\Gamma\left(n+1 / p^{*}\right)}\right]^{p} \sum_{j=0}^{\infty}\left|\sum_{k=0}^{j} \frac{\left(\begin{array}{c}
m+k-1 \\
k
\end{array}\right)}{\left(\begin{array}{c}
m+j \\
j
\end{array}\right)} x_{k}\right|^{p} .
$$


In particular, for $m=n$,

$$
\sum_{j=0}^{\infty}\left|\sum_{k=0}^{j} \frac{\left(\begin{array}{c}
n+j-k-1 \\
j-k
\end{array}\right)}{\left(\begin{array}{c}
n+j \\
j
\end{array}\right)} x_{k}\right|^{p} \leq\left[\frac{\Gamma(n) \Gamma\left(1 / p^{*}\right)}{\Gamma(n-1 / p)}\right]^{p} \sum_{j=0}^{\infty}\left|\sum_{k=0}^{j} \frac{\left(\begin{array}{c}
n+k-1 \\
k
\end{array}\right)}{\left(\begin{array}{c}
n+j \\
j
\end{array}\right)} x_{k}\right|^{p} .
$$

(c) For $n \geq m \geq 0$,

$$
\sum_{j=0}^{\infty}\left|\sum_{k=0}^{j} \frac{\left(\begin{array}{c}
n+j-k-1 \\
j-k
\end{array}\right)}{\left(\begin{array}{c}
n+j \\
j
\end{array}\right)} x_{k}\right|^{p} \leq\left[\frac{\Gamma(n+1) \Gamma\left(m+1 / p^{*}\right)}{\Gamma(m+1) \Gamma\left(n+1 / p^{*}\right)}\right]^{p} \sum_{j=0}^{\infty}\left|\sum_{k=0}^{j} \frac{\left(\begin{array}{c}
m+j-k-1 \\
j-k
\end{array}\right)}{\left(\begin{array}{c}
m+j \\
j
\end{array}\right)} x_{k}\right|^{p} .
$$

In particular, for $n=1, m=0$, we have Hardy's inequality.

See $[4,5]$ for similar inequalities of the above type.

Corollary 3.3 The Hilbert operator $\mathfrak{H}$ has a factorization of the form $\mathfrak{H}=B^{n} C^{n}$, where $B^{n}$ was defined by (3.1).

Proof First we show that

$$
B^{n} \Gamma^{n}=B^{n-1}
$$

In fact,

$$
\begin{aligned}
\left(B^{n} \Gamma^{n}\right)_{i, k} & =\sum_{j=k}^{\infty}\left(\begin{array}{c}
n+j \\
j
\end{array}\right) \beta(i+j+1, n+1) \frac{\left(\begin{array}{c}
n+k-1 \\
k
\end{array}\right)}{\left(\begin{array}{c}
n+j \\
j
\end{array}\right)} \\
& =\left(\begin{array}{c}
n+k-1 \\
k
\end{array}\right) \sum_{j=0}^{\infty} \beta(i+j+k+1, n+1) \\
& =\left(\begin{array}{c}
n+k-1 \\
k
\end{array}\right) \int_{0}^{1} \sum_{j=0}^{\infty} z^{j} z^{i+k}(1-z)^{n} d z \\
& =\left(\begin{array}{c}
n+k-1 \\
k
\end{array}\right) \int_{0}^{1} z^{i+k}(1-z)^{n-1} d z \\
& =\left(\begin{array}{c}
n-1+k \\
k
\end{array}\right) \beta(i+k+1, n)=B_{j, k}^{n-1} .
\end{aligned}
$$

This establishes (3.6). Now, by commutativity of Hausdorff matrices and by (3.6),

$$
B^{n} C^{n}=B^{n} \Gamma^{n} \cdots \Gamma^{1}=B^{n-1} \Gamma^{n-1} \cdots \Gamma^{1} .
$$

Therefore, by induction,

$$
B^{n} C^{n}=B^{1} \Gamma^{1}=B^{0}=\mathfrak{H} .
$$

\section{The mapping $C^{n}: A(p) \rightarrow \ell_{p}$}

We are now ready to study the mappings $C^{n}: A(p) \rightarrow \ell_{p}$. Based on the cases described in Sect. 2, two categories of $A(p)$ spaces are treated below: the Cesàro sequence space $C^{n}(p)$ and the gamma sequence space $\Gamma^{n}(p)$. First, we need the following lemma. 
Lemma 4.1 Let $U$ be a bounded operator on $\ell_{p}$ and $A(p)$ be a matrix domain such that $A(p) \simeq \ell_{p}$. If $T$ has a factorization of the form $T=U A$, then $T$ is a bounded operator from the matrix domain $A(p)$ into $\ell_{p}$ and

$$
\|T\|_{A(p) \rightarrow \ell_{p}}=\|U\|_{\ell_{p} \rightarrow \ell_{p}}
$$

Proof Since $A(p)$ and $\ell_{p}$ are isomorphic, hence

$$
\begin{aligned}
\|T\|_{A(p) \rightarrow \ell_{p}} & =\sup _{x \in A(p)} \frac{\|T x\|_{\ell_{p}}}{\|x\|_{A(p)}}=\sup _{x \in A(p)} \frac{\|U A x\|_{\ell_{p}}}{\|A x\|_{\ell_{p}}} \\
& =\sup _{y \in \ell_{p}} \frac{\|U y\|_{\ell_{p}}}{\|y\|_{\ell_{p}}}=\|U\|_{\ell_{p} \rightarrow \ell_{p} .} .
\end{aligned}
$$

We can follow a similar procedure to obtain the norm of Cesàro matrix from an $A(p)$ space into an $\ell_{p}$ space.

Corollary 4.2 Let $n \geq m \geq 0$. Then $C^{n}: C^{m}(p) \rightarrow \ell_{p}$ is bounded and

$$
\left\|C^{n}\right\|_{C^{m}(p) \rightarrow \ell_{p}}=\frac{\Gamma(n+1) \Gamma\left(m+1 / p^{*}\right)}{\Gamma(m+1) \Gamma\left(n+1 / p^{*}\right)} .
$$

Also, $C^{n}: C(p) \rightarrow \ell_{p}$ is bounded and

$$
\left\|C^{n}\right\|_{C(p) \rightarrow \ell_{p}}=\frac{\Gamma(n+1) \Gamma\left(1+1 / p^{*}\right)}{\Gamma\left(n+1 / p^{*}\right)} .
$$

Proof According to Theorem 3.1 $C^{n}=S^{n, m} C^{m}$, hence Lemma 4.1 finishes the proof.

Corollary 4.3 Let $n \geq m \geq 1$. Then $C^{n}: \Gamma^{m}(p) \rightarrow \ell_{p}$ is bounded and

$$
\left\|C^{n}\right\|_{\Gamma^{m}(p) \rightarrow \ell_{p}}=\frac{(1-1 / m p) \Gamma(n+1) \Gamma\left(1 / p^{*}\right)}{\Gamma\left(n+1 / p^{*}\right)} .
$$

In particular, $C^{n}: C(p) \rightarrow \ell_{p}$ is bounded and

$$
\left\|C^{n}\right\|_{C(p) \rightarrow \ell_{p}}=\frac{\Gamma(n+1) \Gamma\left(1+1 / p^{*}\right)}{\Gamma\left(n+1 / p^{*}\right)} .
$$

Proof According to Theorem 3.1, $C^{n}=R^{n, m} \Gamma^{m}$, hence the result will be obtained from Lemma 4.1.

\section{Acknowledgements}

This manuscript has the only one author, and there is no funding for this research.

Funding

There is no funding for this research.

Availability of data and materials

No data have been used in this study.

\section{Declarations}


Authors' contributions

This manuscript has the only one author and nobody has collaborated in writing that. The author read and approved the final manuscript.

\section{Publisher's Note}

Springer Nature remains neutral with regard to jurisdictional claims in published maps and institutional affiliations.

Received: 10 May 2021 Accepted: 15 October 2021 Published online: 29 October 2021

\section{References}

1. Bennett, G.: Lower bounds for matrices. Linear Algebra Appl. 82, 81-98 (1986)

2. Bennett, G.: Lower bounds for matrices II. Can. J. Math. 44, 54-74 (1992)

3. Bennett, G.: Factorizing the Classical Inequalities. Mem. Amer. Math. Soc., vol. 576 (1996)

4. Bennett, G.: An inequality for Hausdorff means. Houst. J. Math. 25(4), 709-744 (1999)

5. Hardy, G.H.: An inequality for Hausdorff means. J. Lond. Math. Soc. 18, 46-50 (1943)

6. Hardy, G.H.: Divergent Series. Oxford University Press, London (1973)

7. Illkhan, M.: Norms and lower bounds of some matrix operators on Fibonacci weighted difference sequence space. Math. Methods Appl. Sci. 42(16), 5143-5153 (2019)

8. Ng, P.-N., Lee, P.-Y.: Cesàro sequence spaces of non-absolute type. Comment. Math. Prace Mat. 20(2), $429-433$ (1978)

9. Roopaei, H.: Norms of summability and Hausdorff mean matrices on difference sequence spaces. Math. Inequal. Appl. 22(3), 983-987 (2019)

10. Roopaei, H.: Norm of Hilbert operator on sequence spaces. J. Inequal. Appl. 2020, 117 (2020)

11. Roopaei, H.: A study on Copson operator and its associated sequence space. J. Inequal. Appl. 2020, 120 (2020)

12. Roopaei, H.: Factorization of Cesàro and Hilbert matrices based on generalized Cesàro matrix. Linear Multilinear Algebra 68(1), 193-204 (2020)

13. Roopaei, H.: Factorization of the Hilbert matrix based on Cesàro and gamma matrices. Results Math. 75(1), 3 (2020) https://doi.org/10.1007/s00025-019-1129-1

14. Roopaei, H., Başar, F.: On the spaces of Cesaàro absolutely p-summable, null, and convergent sequences. Math. Methods Appl. Sci. 44(5), 3670-3685 (2021)

15. Roopaei, H., Foroutannia, D., Ilkhan, M., Kara, E.E.: Cesàro spaces and norm of operators on these matrix domains. Mediterr. J. Math. 17, 121 (2020)

16. Şengönül, M., Başar, F.: Cesàro sequence spaces of non-absolute type which include the spaces $c_{0}$ and $c$. Soochow J. Math. 31(1), 107-119 (2005)

17. Yaying, T., Hazarika, B., Mohiuddine, S.A., Mursaleen, M.: Estimation of upper bounds of certain matrix operators on binomial weighted sequence spaces. Adv. Oper. Theory 5, 1376-1389 (2020)

18. Yaying, T., Hazarika, B., Mursaleen, M.: Norm of matrix operator on Orlicz-binomial spaces and related operator ideal. J. Math. Comput. Sci. 23, 341-353 (2021)

\section{Submit your manuscript to a SpringerOpen ${ }^{\circ}$ journal and benefit from:}

- Convenient online submission

- Rigorous peer review

- Open access: articles freely available online

- High visibility within the field

- Retaining the copyright to your article

Submit your next manuscript at $\boldsymbol{~ s p r i n g e r o p e n . c o m ~}$ 